\title{
Pratiques
}

Linguistique, littérature, didactique

187-188 | 2020

Enseignement du texte littéraire dans l'espace

francophone: pratiques, formation, recherche

\section{Paroles de formateurs/formatrices d'enseignants du secondaire}

Table ronde $n^{\circ} 2$

Sylviane Ahr et Christine Boutevin

\section{OpenEdition \\ Journals}

\section{Édition électronique}

URL : https://journals.openedition.org/pratiques/9193

DOI : 10.4000/pratiques.9193

ISSN : 2425-2042

\section{Éditeur}

Centre de recherche sur les médiations (CREM)

\section{Référence électronique}

Sylviane Ahr et Christine Boutevin, «Paroles de formateurs/formatrices d'enseignants du

secondaire ", Pratiques [En ligne], 187-188 | 2020, mis en ligne le 12 décembre 2020, consulté le 21

juillet 2021. URL : http://journals.openedition.org/pratiques/9193 ; DOI : https://doi.org/10.4000/

pratiques.9193

Ce document a été généré automatiquement le 21 juillet 2021.

(c) Tous droits réservés 


\section{Paroles de formateurs/formatrices d'enseignants du secondaire}

Table ronde $\mathrm{n}^{\circ} 2$

Sylviane Ahr et Christine Boutevin

1 Dans le prolongement des communications et des discussions fort riches qui ont eu lieu au cours des deux premières journées du colloque, l'équipe Pelas, organisatrice de cette manifestation scientifique, a programmé une table ronde réunissant cinq formateurs d'enseignants du secondaire :

- Françoise Andrieu, professeure formatrice académique (PFA $\left.{ }^{1}\right)$ de lettres auprès des professeurs de lycée professionnel (PLP) de lettres-histoire ou de lettres-langue à l'Institut national supérieur du professorat et de l'éducation (Inspé2) de Bordeaux ;

- Catherine Anglade, PFA de lettres modernes auprès des professeurs de lycée et de collège (PLC) à l'Inspé de Toulouse ;

- Béatrice Gerlaud, PFA de lettres modernes auprès des PLC à l'Inspé de l'académie de Versailles;

- Dominique Ledur, maitre-assistante en didactique du français au sein de la Haute École Galilée - Institut Supérieur de Pédagogie Galilée (ISPG), situé à Bruxelles ;

- Pierre Moinard, maitre de conférences à l'Inspé de Poitiers, professeur agrégé formateur (Prag 3 ) à l'Éspé de l'académie de Versailles au moment du colloque.

2 À partir des expériences professionnelles de chacun, une discussion a été engagée. Il s'est agi d'interroger la formation, initiale et continue, des enseignants à cet objet complexe que sont les approches analytiques des textes littéraires. En effet, les instructions officielles actuelles, nourries des travaux de recherche en didactique de la littérature, recommandent le renouvèlement de ces approches de façon plus ou moins explicite selon les paliers et les filières d'enseignement et selon les aires de la francophonie.

3 En France comme en Belgique et, plus précisément, dans la Fédération WallonieBruxelles, les instructions officielles encouragent la prise en compte de la réception des élèves lecteurs dans les diverses activités liées à l'analyse des textes littéraires. Cette orientation est tout à fait perceptible dans les programmes français actuels du cycle 3 
(fin du primaire et début du secondaire) et, de façon plus implicite, dans les programmes du cycle 4 (trois dernières années du collège) ainsi que dans ceux du lycée professionnel et ceux du lycée général et technologique, entrés en application à la rentrée scolaire 2019. Les référentiels belges demandent aux enseignants de favoriser un questionnement sur ce que l'élève ressent, observe et interprète. Dans tous les cas et selon des éclairages épistémologiques et didactiques plus ou moins précis, il est recommandé aux professeurs de porter une attention égale à l'expérience subjective des élèves lecteurs et à l'acquisition de connaissances langagières, littéraires et culturelles, l'enjeu étant de former des lecteurs interprètes. Les échanges, dont nous proposons ici une synthèse, ont donc concerné la prise en compte de cette double recommandation institutionnelle par les (futurs) professeurs dans le cadre de leur enseignement par les formateurs eux-mêmes lors des séances de formation initiale ou continue, et cela pour l'ensemble des paliers de l'enseignement secondaire.

\section{Acquisition de connaissances langagières, littéraires et culturelles et prise en compte des expériences subjectives des élèves lecteurs : une articulation complexe}

4 P. Moinard reconnait tout d'abord que la demande institutionnelle quant à la prise en compte des expériences subjectives dans les approches analytiques des textes littéraires semble bien reçue par les professeurs en formation continue. Certains voient dans les verbalisations des réceptions des élèves les marques d'un engagement dans ces approches. C. Anglade ajoute même que les stagiaires connaissent tous désormais les théories de la réception ainsi que les textes de référence sur la lecture littéraire et qu'ils admettent assez volontiers la nécessaire prise en compte de l'élève comme sujet lecteur. Elle constate que ces professeurs en formation manifestent un intérêt certain, par exemple, pour le journal du lecteur et qu'ils sont souvent curieux d'expérimenter des pratiques variées et nouvelles qui mobiliseraient les élèves et leur donneraient le plaisir de lire.

5 Cependant, tous les formateurs évoquent les difficultés que représente la nécessité d'articuler l'acquisition de connaissances et l'expression de la subjectivité des élèves. Ainsi B. Gerlaud explique que les jeunes collègues commencent par juxtaposer des temps centrés sur les connaissances langagières, l'étude de la langue étant alors mise au service de la lecture, et des temps consacrés aux connaissances culturelles et littéraires pour contextualiser les textes et les œuvres étudiés en classe. En France comme en Belgique, selon les formateurs, soit les stagiaires laissent assez peu de place à l'expression des expériences subjectives des élèves lecteurs ou accueillent avec réserve leurs réceptions affectives et/ou axiologiques des textes, soit ils se déclarent euxmêmes démunis devant les difficultés à susciter des réactions suffisamment claires ou exploitables. Pour P. Moinard, de manière plus générale, les professeurs en formation continue ne voient pas comment articuler les verbalisations de lectures singulières et les analyses de textes souvent envisagées uniquement comme le développement du commentaire professoral. Selon B. Gerlaud, les stagiaires PLC s'efforcent de "coller " aux programmes. F. Andrieu fait le même constat en précisant que les stagiaires PLP prennent les programmes au pied de la lettre : ils privilégient une lecture linéaire des 
textes, qu'ils souhaitent exhaustive de peur «d'oublier » quelque chose d'important. Elle ajoute qu'ils n'ont pas la maitrise ni le recul nécessaires pour faire des choix.

6 Les formatrices françaises donnent à ces constats certaines explications liées au parcours universitaire des stagiaires et à leur conception du métier. F. Andrieu pense que la majorité des stagiaires PLP a des représentations du cours de français qui datent de leurs études secondaires: nombreux sont ceux qui n'ont pas reçu de formation universitaire en lettres. Selon C. Anglade, les pratiques des stagiaires PLC, pour ce qui concerne l'enseignement de la lecture des textes littéraires, correspondent parfois à des représentations du métier dictées par leur propre expérience de bons élèves. La formatrice précise que cela peut entrainer la crainte de ne pas suffisamment apporter de savoirs savants aux élèves et se traduit par le souci de garder des traces pérennes du cours (synthèses dictées dans les classeurs et réponses rédigées aux questions posées). Selon D. Ledur, cette difficulté à accueillir les dimensions affectives de la lecture des élèves peut être due à un sentiment d'insécurité de la part des stagiaires. En effet, ces derniers se demandent comment évaluer la pertinence des ressentis exprimés par les élèves, s'il faut en garder trace, quel statut leur donner ou comment les intégrer dans la construction collective du sens du texte. Enfin, certaines réalités ne permettraient pas aux étudiants stagiaires de donner une place aux subjectivités singulières, car, comme ils l'ont confié à B. Gerlaud, une année scolaire passe vite, les classes sont hétérogènes, les élèves n'ont pas toujours les moyens langagiers (lexicaux surtout) de mettre en mots leur réception des textes; certains ne disent rien parce qu'ils pensent que ce qu'ils auraient à dire ne peut pas intéresser, en particulier, un enseignant ; d'autres ont été habitués, culturellement, à se taire (souvent des filles). À ces difficultés éprouvées et exprimées par les stagiaires s'ajoutent celles liées à la gestion de la classe, comme le constate C.Anglade: elles empêchent les jeunes collègues d'être à l'écoute des expériences de lecture subjective des élèves.

7 Quelles conséquences ces divers «obstacles» déclarés ont-ils sur les pratiques d'enseignement de la littérature et de sa lecture et, plus particulièrement, sur les approches analytiques des textes littéraires en début de carrière? C. Anglade détaille ainsi ce qu'elle a pu observer dans les classes des stagiaires qu'elle accompagne :

Si l'on considère les moments clés des séances de lecture, le temps de prise en compte du lecteur est généralement dévolu aux quinze premières minutes et souvent de façon assez artificielle : nombreux sont ceux qui se soucient de préparer une "question de réception " pour ménager un temps où l'élève réfléchit seul et livre sa première compréhension ou ses «impressions" sur le texte. [...] Le deuxième temps de la séance de lecture est encore presque toujours consacré à des questions très dirigées auxquelles les élèves répondent seuls ou en groupes afin de valider un sens préalablement construit par le professeur. Pour autant, les savoirs ne sont pas toujours clairement construits ou identifiés.

B. Gerlaud dresse les mêmes constats en précisant que l'approche d'un texte se fait souvent par les impressions de lecture demandées aux élèves à l'initiale du cours et dont les professeurs stagiaires ne s'emparent que si celles-ci correspondent aux analyses détaillées préparées en amont, les impressions de lecture étant donc éludées voire rejetées si elles ne correspondent pas aux axes de lecture prévus par le professeur. D. Ledur observe cette même attitude de la part des jeunes collègues belges qui, face à des interrogations non résolues et, de fait, à la source du sentiment d'insécurité évoqué plus haut, réorientent fréquemment l'échange à propos du texte vers le repérage de caractéristiques génériques, littéraires, langagières. Finalement, comme P. Moinard l'explique de manière synthétique, « la séance de lecture analytique 
semble relever de deux démarches juxtaposées et non reliées : l'accueil des affects des élèves lecteurs est effectué en parallèle de la description des effets sur lesquels le professeur veut mettre l'accent $»$.

Ce premier échange entre formateurs met au jour une double tendance observée dans les pratiques, déclarées mais aussi effectives, des enseignants stagiaires ou expérimentés: conformément aux recommandations institutionnelles, ceux-ci accueillent volontiers, dans un premier temps, les lectures subjectives des élèves; cependant, grande est leur difficulté à articuler ces lectures singulières - et souvent actualisantes - aux objectifs d'apprentissage qu'ils se sont fixés et à la lecture experte à laquelle ils se sont eux-mêmes livrés en amont de la séance, lecture qu'ils estiment devoir être commune à tous. Ce double constat conduit à interroger les actions de formation susceptibles de favoriser cette articulation complexe qu'il convient de ménager entre la prise en compte des expériences subjectives des élèves lecteurs et l'acquisition de connaissances langagières, littéraires et culturelles. C'est le deuxième point sur lequel les formateurs ont été invités à discuter dans le cadre de cette table ronde.

\section{Adaptation de la formation à cette articulation complexe}

10 Il semble y avoir consensus quant à la nécessité de placer tout d'abord les stagiaires, quel que soit leur parcours universitaire et/ou professionnel et que ce soit en formation initiale ou continue, dans la situation de lecteurs avant même d'examiner avec eux les modalités selon lesquelles l'institution leur recommande de mettre en œuvre une approche analytique des textes littéraires dans les classes du secondaire. En effet, Pour B.Gerlaud, la priorité consiste à faire évoluer les représentations des stagiaires pour ce qui concerne la réception subjective des textes. Il leur est donc demandé de se positionner tout d'abord comme lecteurs et non comme enseignants de lettres, en quelque sorte de "recevoir les textes en tant que sujets lecteurs ", même si, en tant que PLC, ils sont des lecteurs avertis, « amoureux de la littérature ». Et c'est une situation analogue que F. Andrieu propose aux stagiaires PLP, qui majoritairement n'ont pas bénéficié d'une formation universitaire littéraire et qui, par conséquent, n'ont que leurs souvenirs scolaires comme modèle d'une approche analytique des textes littéraires. Il s'agit par conséquent de placer les stagiaires en situation «d'élèves » dans le cadre de la lecture analytique d'un texte afin de les amener à faire eux-mêmes l'expérience de la lecture littéraire, c'est-à-dire de ce mouvement dialectique entre lecture subjective et lecture objective, entre participation et distanciation, entre expérience sensible et examen critique des textes littéraires. Selon D. Ledur, demander aux stagiaires de verbaliser oralement leur expérience de lecture, leur faire produire des écrits de réception - des "écrits d'appropriation", comme les programmes français du lycée (professionnel, général et technologique) mis en application à la rentrée 2019 le prescrivent - les amènent à conceptualiser les différents types de posture du lecteur que ces tâches induisent. En outre, afin que les (futurs) enseignants admettent plus aisément qu'un même texte littéraire, en raison de sa polysémie consubstantielle, peut se prêter à diverses approches analytiques, les formateurs proposent la confrontation de ces écrits de réception produits par les stagiaires, voire par les élèves. Le but est alors de dégager, comme le précise B. Gerlaud, 
les points communs et les divergences entre ces divers écrits tant sur le plan du sens que sur celui des formes linguistiques et/ou non linguistiques. Pour D. Ledur, cette confrontation entre les écrits de réception produits par les élèves et ceux produits par les stagiaires permet d'initier ces derniers aux situations professionnelles au cours desquelles ils devront gérer non seulement la diversité des expériences de lecture mais également articuler cette pluralité de lectures à leur propre réception du texte ainsi qu'aux objectifs d'apprentissage visés. En effet, comme l'explique F. Andrieu, ces mises en situation de lecture personnelle puis de confrontation permettent aux stagiaires d'examiner certains gestes professionnels, tel celui du tissage, et, par là même, d'apprendre à gérer l'équilibre entre leur parole et celle des élèves. P. Moinard confirme que ces mises en situation conduisent les professeurs en formation, en l'occurrence en formation continue, «de vivre une trajectoire réflexive reliant le partage des réactions à la co-élaboration d'un projet de lecture analytique ». La confrontation des écrits de réception qu'ils ont produits amène les enseignants à élaborer collectivement un questionnement sur le texte et à percevoir de la sorte comment il est possible d'articuler les verbalisations de lectures singulières et l'analyse du texte.

11 Selon C. Anglade, il est important également que les stagiaires puissent réfléchir sur la constitution des corpus et sur leur problématisation dans la mesure où les programmes français du collège, en application depuis la rentrée 2016, assignent à l'enseignement de la littérature des enjeux à la fois littéraires et de formation personnelle. La formatrice explique :

Il s'agit de se demander comment les textes retenus peuvent avoir quelque chose à dire aux élèves d'aujourd'hui et poser des questions existentielles qui les intéressent en plus des questions littéraires. La recherche de la problématique est ainsi un levier indispensable pour amener les stagiaires à s'interroger sur les raisons pour lesquelles ils ont rassemblé des textes en corpus et sur le projet de lecture qu'ils souhaitent conduire pour impliquer les élèves en tant que lecteurs.

Conduire les stagiaires à concevoir un projet de lecture qui tende conjointement à « impliquer les élèves en tant que lecteurs » et à développer les connaissances ainsi que les compétences attendues chez ces mêmes élèves à chaque palier de la scolarité est un objectif prioritaire assigné à la formation. Force est d'admettre cependant que les textes officiels cadrant l'approche analytique des textes littéraires dans les différents paliers de la scolarité reposent sur des conceptions sensiblement différentes des enjeux et des modalités de cette approche. Il a donc été demandé aux formateurs de préciser comment, dans le cadre de leurs actions de formation et en fonction des structures universitaires desquelles ils dépendent respectivement, était prise en compte la progression curriculaire des élèves lecteurs.

\section{Progression curriculaire en matière d'approches analytiques des textes littéraires}

13 Pour B. Gerlaud, il s'avère indispensable de faire évoluer les conceptions des lauréats des concours : ceux-ci pensent non seulement que l'on étudie la langue et acquiert les outils d'analyse au collège et que l'on apprend à les utiliser au lycée, mais aussi que certaines activités, notamment "ludiques", sont réservées aux collégiens, d'autres, «plus sérieuses», aux lycéens. Afin que les stagiaires - en formation initiale ou 
continue - s'interrogent sur la progression des apprentissages à ménager au fil de la scolarité, les formateurs proposent des séances au cours desquelles les (futurs) enseignants confrontent des approches analytiques d'un même texte réalisées ou à réaliser avec des élèves de niveaux différents ainsi que des écrits de réception produits par ces derniers suite à la lecture d'un même texte, que celui-ci appartienne à la littérature classique ou contemporaine pour adultes ou bien à la littérature de jeunesse. En effet, comme D. Ledur le souligne, les œuvres de littérature de jeunesse requièrent aujourd'hui « un lecteur actif, qui adhère et se méfie, qui prend plaisir à résoudre les énigmes narratives, à déceler les liens intertextuels ». Il convient dès lors de rappeler aux enseignants en formation qu' 'il importe de ne pas aller du simple vers le complexe, mais de proposer dès le début des textes qui sollicitent l'activité interprétative du lecteur ", ce qu'un grand nombre d'œuvres de littérature de jeunesse favorisent.

14 Selon P. Moinard, de telles démarches de formation conduisent les professeurs à "désindexer la lecture analytique de l'écriture de commentaires littéraires ou composés ", cette écriture étant généralement l'enjeu exclusif assigné aux séances de lecture par les enseignants de fin de collège et de lycée. C. Anglade explique aussi que, pour favoriser la prise en compte de la progression curriculaire entre la fin du cycle 4 (collège) et le lycée, les stagiaires en collège et en lycée sont regroupés dans certains modules de formation initiale. Ainsi peuvent-ils : croiser leurs expériences de terrain, en travaillant par exemple sur des albums; et confronter les modalités selon lesquelles ils élaborent leurs corpus, leurs séquences ou parcours d'enseignement et questionnent les textes. Afin d'assurer la liaison au sein du cycle 3 (école élémentaire/collège), cette même démarche est aussi mise en œuvre auprès des stagiaires du premier degré et ceux du second degré. De même, F. Andrieu estime nécessaire d'organiser des rencontres entre stagiaires PLC et stagiaires PLP, ces derniers devant être informés des enseignements dispensés par leurs collègues en amont du lycée professionnel. En effet, selon la formatrice française, nombreux sont les stagiaires « qui ont tendance à oublier que leurs élèves ont un passé en arrivant au lycée professionnel ", même si ce passé scolaire se signale davantage par des échecs que par des réussites.

15 Enfin, comme D. Ledur le rappelle, «la lecture est avant tout une tâche globale, complexe, difficile à morceler ». Son apprentissage doit donc se réaliser selon une progression spiralaire, ce qui, de toute évidence, requiert un travail collaboratif de la part des formateurs des différents degrés d'enseignement.

16 Cette synthèse des échanges entre formateurs montre que la formation, initiale et continue, des professeurs du secondaire se nourrit, en France comme en Belgique, des travaux de recherche en didactique de la littérature, qui tendent à favoriser le renouvèlement des approches analytiques des textes littéraires, même si ces évolutions sont plus ou moins marquées dans les programmes prescrits. Cependant, comme le soulignent les participants à cette table ronde, des difficultés demeurent: outre certaines résistances conceptuelles, l'articulation entre la prise en compte des expériences subjectives des élèves lecteurs et l'acquisition des connaissances langagières, littéraires et culturelles reste pragmatiquement complexe, ce qui place au second plan toute réflexion portant sur la formation des lecteurs selon une progression curriculaire. Les actions de formation évoquées dans cette brève synthèse visent à lever ces obstacles. Puisse-t-elle offrir aux formateurs et aux chercheurs quelques pistes à 
approfondir dans le cadre de travaux collaboratifs sur cet objet complexe que sont les approches analytiques des textes littéraires!

\section{BIBLIOGRAPHIE}

Ministère de l'Éducation nationale de la Jeunesse et des Sports (2016). « Formation initiale et continue », Bulletin officiel n ${ }^{\circ} 38$ du 20 octobre 2016. En ligne : https://www.education.gouv.fr/ bo/16/Hebdo38/MENH1605074C.htm?cid_bo=107464.

\section{NOTES}

1. En France, les PFA «accomplissent à la fois une mission d'enseignement ou d'éducation auprès d'élèves, en leur qualité d'enseignant ou de CPE, et une mission de formation auprès d'adultes, en leur qualité de formateur " (Ministère de l'Éducation nationale, de la Jeunesse et des Sports). Les PFA réalisent donc la moitié de leur service dans un collège ou un lycée, l'autre moitié dans un Inspé.

2. En France, les Écoles supérieures du professorat et de l'éducation (Éspé) ont été renommées Instituts nationaux supérieurs du professorat et de l'éducation (Inspé) le $1^{\mathrm{er}}$ septembre 2019 par le ministère de l'Éducation nationale, de l'Enseignement supérieur et de la Recherche.

3. Un Prag est un professeur agrégé qui exerce à temps complet dans le supérieur, en l'occurrence dans un Inspé.

\section{RÉSUMÉS}

Les communications et discussions qui ont eu lieu au cours des deux premières journées du colloque - et dont une partie est rassemblée dans le présent numéro de Pratiques - ont été prolongées par une table ronde réunissant quatre formateurs français et une formatrice belge d'enseignants du secondaire. À partir des expériences professionnelles de chacun a été engagée une discussion autour de la formation, initiale et continue, des enseignants à cet objet fort complexe que sont les approches analytiques des textes littéraires, approches dont les instructions officielles actuelles, nourries des travaux de recherche en didactique de la littérature, recommandent le renouvèlement de façon plus ou moins explicite selon les paliers et les filières d'enseignement et selon les aires de la francophonie. Dans tous les cas et selon des éclairages épistémologiques et didactiques plus ou moins précis, il est en effet conseillé aux professeurs de porter une attention égale à l'expérience subjective des élèves lecteurs et à 
l'acquisition de connaissances langagières, littéraires et culturelles, l'enjeu étant de former des lecteurs interprètes. Les échanges, dont il est proposé ici une synthèse, ont donc concerné la prise en compte de cette double recommandation institutionnelle par les (futurs) professeurs dans le cadre de leur enseignement ainsi que par les formateurs eux-mêmes lors des séances de formation initiale ou continue, et cela pour l'ensemble des paliers de l'enseignement secondaire.

The presentations and discussions that took place during the first two days of the symposium some of which are collected in this issue of Pratiques - were followed by a round table with four French trainers and a Belgian secondary school teacher trainer. Based on the professional experiences of each participant, a discussion was held on the initial and ongoing training of teachers in the complex subject of analytical approaches to literary texts, approaches whose current official instructions, supported by research on the didactics of literature, recommend renewal in a more or less explicit manner depending on the levels and streams of instruction and the areas of the Francophonie. In all cases and according to more or less precise epistemological and didactic insights, teachers are in fact advised to pay equal attention to the subjective experience of student readers and to the acquisition of linguistic, literary and cultural knowledge, the challenge being to train interpreter-readers. The exchanges, which are summed up here, therefore concerned the consideration of this twofold institutional recommendation by

(future) instructors in their teaching and by the trainers themselves during initial or continuing training sessions, for all levels of secondary education.

\section{INDEX}

Mots-clés : Formation des enseignants, formation des lecteurs interprètes, progression curriculaire

Keywords : Teacher training, training of reader-interpreters, curriculum progression

\section{AUTEURS}

\section{SYLVIANE AHR}

Université de Toulouse-Jean Jaurès, UT2J/LLA-Créatis, F-31058 Toulouse, France

\section{CHRISTINE BOUTEVIN}

Université de Montpellier, Lirdef, F-34000 Montpellier, France 